\title{
A comparative study of multiple neural network for detection of COVID-19 on chest X-ray
}

\author{
Anis Shazia ${ }^{1}$, Tan Zi Xuan ${ }^{1}$, Joon Huang Chuah², Juliana Usman', Pengjiang Qian ${ }^{3}$ and Khin Wee Lai ${ }^{1 *}$ (D)
}

\author{
* Correspondence: lai.khinwee@um. \\ edu.my \\ ${ }^{1}$ Department of Biomedical \\ Engineering, Faculty of Engineering, \\ Universiti Malaya, 50603 Kuala \\ Lumpur, Malaysia \\ Full list of author information is \\ available at the end of the article
}

\begin{abstract}
Coronavirus disease of 2019 or COVID-19 is a rapidly spreading viral infection that has affected millions all over the world. With its rapid spread and increasing numbers, it is becoming overwhelming for the healthcare workers to rapidly diagnose the condition and contain it from spreading. Hence it has become a necessity to automate the diagnostic procedure. This will improve the work efficiency as well as keep the healthcare workers safe from getting exposed to the virus. Medical image analysis is one of the rising research areas that can tackle this issue with higher accuracy. This paper conducts a comparative study of the use of the recent deep learning models (VGG16, VGG19, DenseNet121, Inception-ResNet-V2, InceptionV3, Resnet50, and Xception) to deal with the detection and classification of coronavirus pneumonia from pneumonia cases. This study uses 7165 chest $X$-ray images of COVID-19 (1536) and pneumonia (5629) patients. Confusion metrics and performance metrics were used to analyze each model. Results show DenseNet121 (99.48\% of accuracy) showed better performance when compared with the other models in this study.
\end{abstract}

Keywords: Artificial neural networks, Deep learning, Transfer learning, Multi-task learning, COVID-19, Classification, DenseNet121

\section{Springer Open}

\section{Introduction}

The novel coronavirus of 2019, or simply known as the COVID-19, affects the respiratory tracts and the lungs leading to severe cases of pneumonia. The usual symptoms include fever, dry hack cough, body ache, and loss of taste or smell. In extreme cases, the patient may experience shortness of breath and multiple organ failure and may lead to fatality (https://www.worldometers.info/coronavirus/). While the world pharmaceutical companies are trying to develop vaccination to prevent the spread of this pandemic, the current medical practice to control the spread of COVID-19 is focused on early detection and isolation of the patient. The current gold standard for COVID-19 detection is the real-time reverse transcription-polymerase chain reaction (RT-PCR), where the short sequences of DNA or RNA are reproduced or amplified and analyzed [1]. Fang et al. [2]

(c) The Author(s). 2021 Open Access This article is licensed under a Creative Commons Attribution 4.0 International License, which permits use, sharing, adaptation, distribution and reproduction in any medium or format, as long as you give appropriate credit to the original author(s) and the source, provide a link to the Creative Commons licence, and indicate if changes were made. The images or other third party material in this article are included in the article's Creative Commons licence, unless indicated otherwise in a credit line to the material. If material is not included in the article's Creative Commons licence and your intended use is not permitted by statutory regulation or exceeds the permitted use, you will need to obtain permission directly from the copyright holder. To view a copy of this licence, visit http://creativecommons.org/licenses/by/4.0/. 
reported that the RT-PCR testing has a low sensitivity of $71 \%$ while Williams et al. [3] reported that the sensitivity of a single RT-PCR test in hospitalized patients is $82.2 \%$.

Perceiving the limitations of RT-PCR, there is a need for cross-verification examination by using radiological images. Chest radiography, particularly chest X-ray, is one of the most frequently performed diagnostic examinations even in underdeveloped areas. Radiographic scanning was proposed to detect pathological effects of COVID-19 by examining chest radiological images of lungs of patients [4]. Several studies have shown that changes in chest radiography images such as X-ray and CT scan were noticed even before the appearance of clinical features of COVID-19 [5]. Interpretation of chest X-ray (CXR) and CT scans have widely been done by radiologists to find some visual indicators for COVID-19 infection as an alternative method for rapid screening of infected patients. For early-stage COVID-19 on CXR, peripheral ground-glass opacities are observed which progresses to consolidations at later stages [6,7]. Since the studies have shown that the abnormalities caused by COVID-19 are visible in chest X-rays, these abnormalities, especially the opacities, are further used to detect COVID-19.

Radiological COVID-19 detection also experiences challenges due to its similar nature and appearance with viral pneumonia radiographs. It requires medical experts to identify the specific radiographic markers to distinguish between the two conditions. With an enormous number of COVID-19 cases suspected daily, it is difficult to assign enough time and resources to individual radiographs. This discrepancy between the available experts and the need of the human expertise has promoted automation and machine learning to fill this much-needed gap [8]. Over the last year, scientists and researchers are unitedly working to automate the detection methods and provide intelligent machines that can easily distinguish infectious COVID-19 cases from other similar appearing cases. This study is conducted to explore these state-of-the-art techniques that have shown promising result and compare it with the same parameters and datasets to identify the best DL model for COVID-19 detection.

\section{Related work}

Jain et al. [9] implemented ResNet-101 in the classification of COVID-19 and viral pneumonia, achieving an accuracy of $97.78 \%$. Che Azemin et al. [10] used pretrained ResNet-101 to detect COVID-19 in CXR with an accuracy of $71.9 \%$ as their training dataset was based on airspace opacity instead of confirmed COVID-19 cases. Ismael et al. [11] also used ResNet-50 architecture but only for feature extraction. The extracted features were classified using an SVM classifier with the Linear kernel function and produced high accuracy of 94.7\%. Makris et al. [12] fine-tuned several CNN models and compared their performances in classifying COVID-19, pneumonia, and normal images. VGG16 turned out to have the best performance with an overall accuracy of $95.88 \%$ in their study. Abbas et al. [13] proposed a new method to classify COVID-19, SARS, and normal CXR which is called DeTraC (stands for Decompose, Transfer, and Compose). It is done by adding a class decomposition layer to the pretrained models that can partition each image class into sub-classes, but assemble back during prediction. By using VGG19 with the DeTraC approach, the model has achieved a classification accuracy of 
93.1\%. Asif et al. [7] trained InceptionV3 using transfer learning techniques to distinguish COVID-19 from viral pneumonia and normal CXR and obtained an accuracy of 98\%. Inspired by DarkNet architecture, Ozturk et al. [14] developed a deep learning network named DarkCovidNet for automated COVID-19 diagnosis. The model achieved an accuracy of $98.08 \%$ for binary (COVID-19 and normal) and 87.02\% for multiclass (COVID-19, pneumonia, and normal) classification. Shelke et al. [15] worked in the segregation of COVID-19 and normal pneumonia using DenseNet-161 and achieved an accuracy of 98.9\%. Minaee et al. [16] fine-tuned 4 pretrained networks (ResNet18, ResNey50, SqueezeNet, and DenseNet-121) and compared their performance. Different cut-off thresholds for probability score were experimented in this study. SqueezNet turned out to be the best model with a sensitivity of $98 \%$ and a specificity of $92.9 \%$. Das et al. [17] have developed a new model with a weighted average ensembling method; the model comprises of three pre-trained CNN models-DenseNet201, Resnet50V2, and InceptionV3. This approach has achieved an accuracy of $95.7 \%$ and a sensitivity of $98 \%$ in the classification of positive and negative COVID-19 cases. Ridhi et al. [18] proposed a new method to classify COVID-19, pneumonia, and normal CXR by using stacked of DenseNet and GoogleNet as feature extractor, and then the features were classified by the ensemble of XGB, RF, and SVM classifiers. The classification accuracy obtained in this study is $91.7 \%$. Gupta et al. [19] proposed an integrated stacked deep convolution network called InstaCovNet-19 which makes use of InceptionV3, NASNet, Xception, MobileNetV2, and ResNet101. The proposed model achieved an accuracy of $99.53 \%$ in binary (COVID-19 vs non-COVID-19) classification and an accuracy of $99.08 \%$ in 3-class (COVID-19, pneumonia, normal) classification. A 22layer CNN architecture was proposed by Hussain et al. [20] which achieved a classification accuracy of $99.1 \%, 94.2 \%$, and $91.2 \%$ for binary, 3-class, and 4-class classification, respectively. Canayaz et al. [21] developed a model called MH-COVIDNet that used VGG19 as a feature extractor and BPSO meta-heuristic algorithm (MH algorithm) for feature selection. This approach obtained a classification accuracy of 99.38\%. Khuzani et al. [22] performed feature extraction using different techniques such as Texture, FFT, Wavelet, GLCM, and GLDM. In the study, a multilayer network was created with 2 hidden layers of 128 and 16 neurons and a final classifier. The 3-class classification (COVID-19, pneumonia, and normal) has achieved an accuracy of $94 \%$.

Table 1 List of databases used in this study

\begin{tabular}{llll}
\hline & Dataset & COVID-19 & Pneumonia \\
\hline 1 & CoronaHack-Chest X-Ray-Dataset [28] & 19 & 4118 \\
2 & Covid_Data_GradientCresent [29] & 69 & 158 \\
3 & Covid-19 Radiography Database [30] & 1143 & 1345 \\
4 & Covid-chestxray-dataset [31] & 142 & - \\
5 & Figure1-COVID-chestxray-dataset [32] & 35 & - \\
6 & Actualmed-COVID-chestxray-dataset [33] & 58 & - \\
7 & COVID-19-X-ray-10000-images [34] & 70 & 8 \\
8 & ChestXRay2017 [35] & - & 5629 \\
\hline
\end{tabular}


Table 2 Data for training, validation, and testing

\begin{tabular}{lll}
\hline & COVID-19 & Pneumonia \\
\hline Training & 1101 & 1104 \\
Validation & 276 & 276 \\
Testing & 156 & 4249 \\
\hline
\end{tabular}

From the above researches, it is observed that identification of the novel coronavirus on radiological images using deep learning techniques has the potential to reduce the pressure on radiologists. However, with various researchers using different deep learning methods, it is unclear which model provides the best result. Therefore, this study compares various deep learning models that have given impressive results in COVID-19 identification. In this study, we have fine-tuned existing models (VGG16, VGG19, DenseNet121, Inception-ResNet-V2, InceptionV3, Resnet50, and Xception) based on our classification requirements. These models have shown remarkable results in pneumonia detection [23-25] and have also been showing promising results with COVID-19 [11, 26, 27] classification. Hence, in this study, we have compared them based on the same data and variables to determine the best model to distinguish COVID-19 X-ray from pneumonia. The models have been trained and tested on COVID-19 and pneumonia CXR images from multiple datasets to avoid any biases. The models are then compared based on their performance metrics and computational time taken. The results are carefully analyzed, and the best model is chosen for this binary classification.

\section{Materials and methods}

\subsection{Dataset}

Due to the limitation of publicly available COVID-19 data, we have complied with multiple databases for this study. All images collected for pneumonia and COVID-19 are from publicly available datasets. Table 1 tabulates the various databases and the number of images adopted from them; similar images were eliminated. A total of 1536 COVID-19 and 5629 pneumonia images were used for training, validation, and testing of the models. The images collected from these databases were of various dimensions, which was resized to $224 \times 224$ pixels.

From the total samples of COVID-19, 10\% of samples was randomly selected for testing. The remaining sample was split into $80 \%$ for training and $20 \%$ for validation.

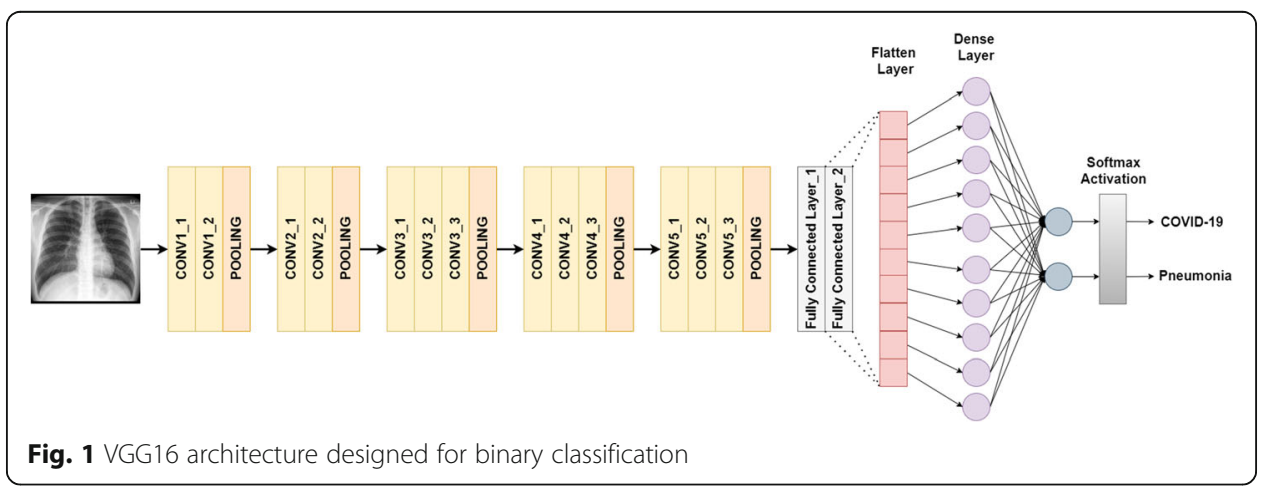




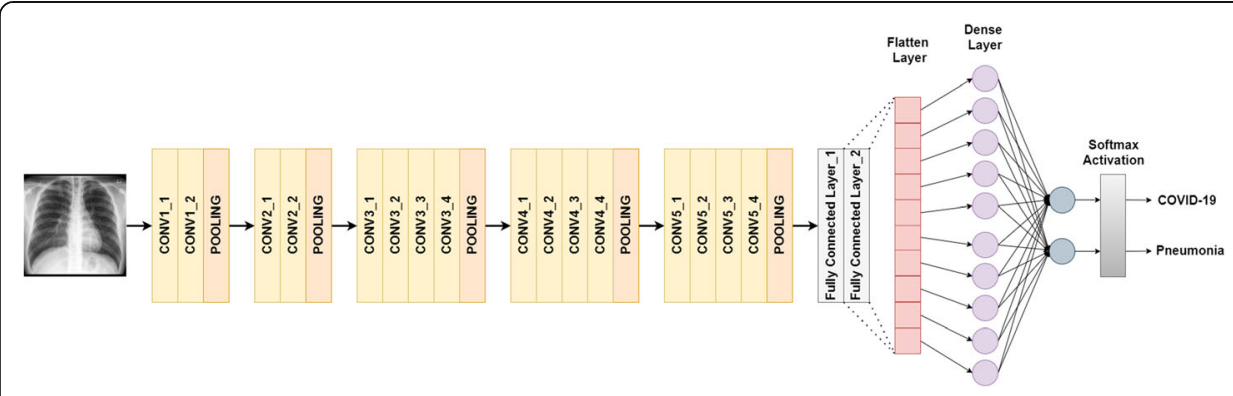

Fig. 2 VGG19 architecture designed for binary classification

Similarly, a balanced dataset was obtained by randomly selecting a similar number of samples for training and validation of pneumonia and splitting them $80 \%$ for training and $20 \%$ for validation. The remaining samples were used for testing. Table 2 tabulates the total images used in each class used for training, validation, and testing. The training and validation tests were balanced to obtain a better result and to avoid overfitting to the majority class that is the pneumonia cases. A balanced training set has been observed to give the highest accuracy regardless of the instances in the test dataset [36]. Also, the models were exposed to images from various databases to avoid any biases towards a database. Also, the imbalance between the two test sets was done to imitate a real-life environment where a number of cases are not balanced and are not from one particular source.

\subsection{Transfer learning approach}

There are two types of transfer learning in the context of deep learning, which are feature extraction and fine-tuning. In the feature extraction technique, a pretrained model on some standard dataset such as ImageNet is used, but the top layer, which is used for classification purpose, will be removed. Then on top of the pretrained model, it trains a new classifier to perform classification. The pretrained model without the top classifier is treated as an arbitrary feature extractor in order to extract useful features from the new dataset. In the second approach which is fine-tuning, the pretrained model weights are treated as the initial values for the new training, and they are updated and adjusted in the training process. In this case, the weights are fine-tuned from generic feature

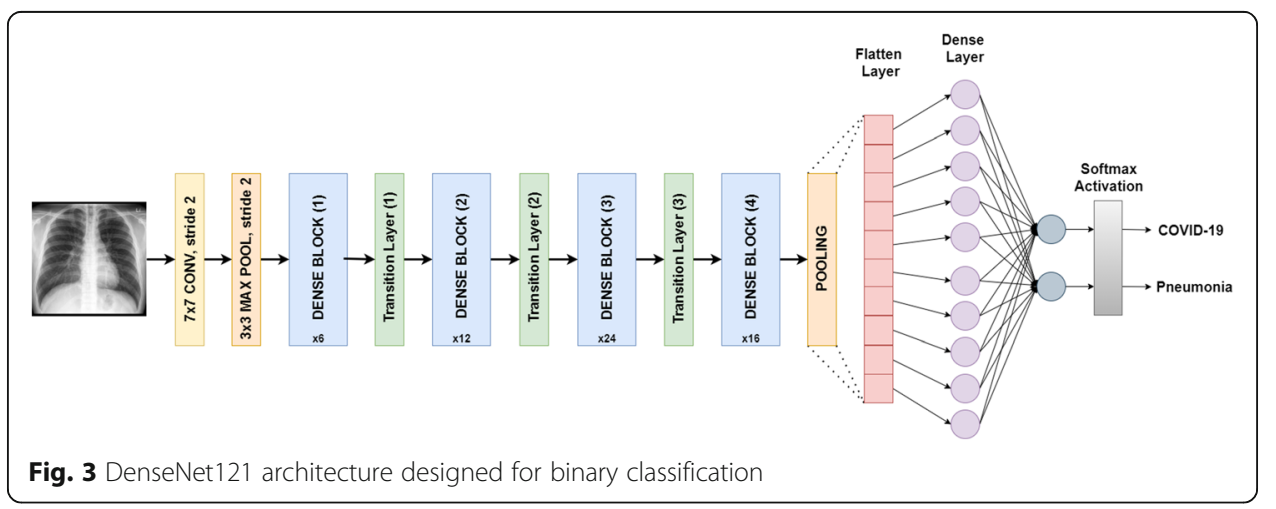




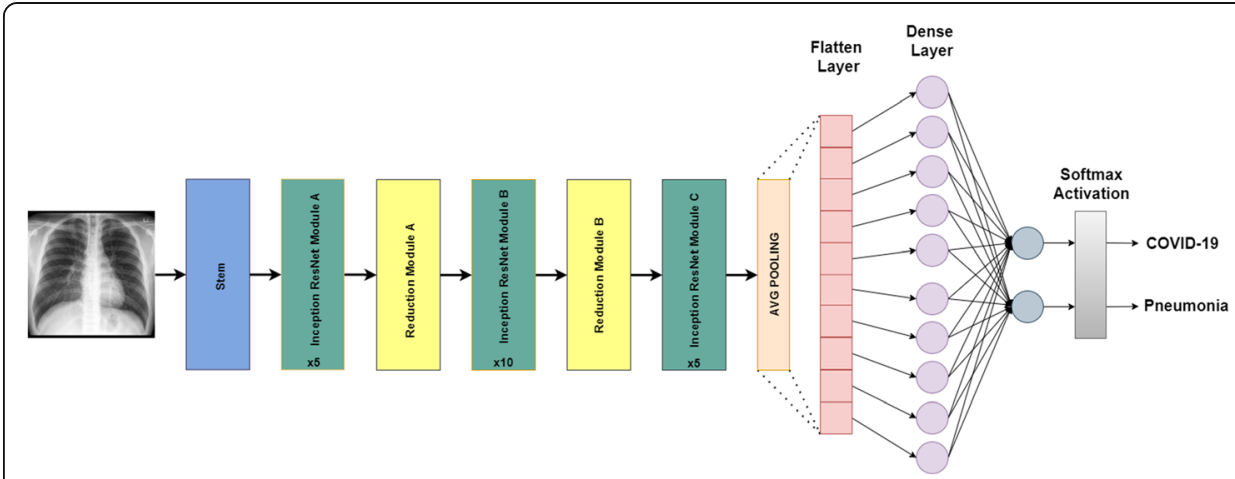

Fig. 4 Inception-ResNet-V2 architecture designed for binary classification

maps to specific features associated with the new dataset. The goal of fine-tuning is about adapting the generic features to a given task rather than overwriting the generic learning.

For this study, a transfer learning approach was adopted and pre-trained weights from ImageNet were used to compensate for the small training data set. With transfer learning, the models were prevented from overfitting due to the small data set. In this study, we fine-tuned the last layer of seven state-of-the-art deep learning modelsVGG16, VGG19, DenseNet12, Inception-ResNet-V2, InceptionV3, ResNet50, and Xception-while using the pre-trained model as a feature extractor. To fine-tune these models for binary classification, the last set of layers which consists of fully-connected layers along with softmax activation function were replaced with a flatten layer, which converts the data from the previous layer to a giant 1-dimensional tensor. A dropout of 0.5 was added for regularization, and lastly, a dense layer was added which applied softmax activation on previous layers and produce two outputs of probability for "COVID19 " and "pneumonia" classes. The next section will briefly discuss the architecture of these models and how they are used for this binary classification.

\subsubsection{VGG16}

The input of VGG16 is of fixed size $224 \times 224$ RGB image. It consists of 16 layers which include 13 convolutional layers and 3 fully connected layers, including maxpooling to reduce the volume size and softmax classifier following the last fully

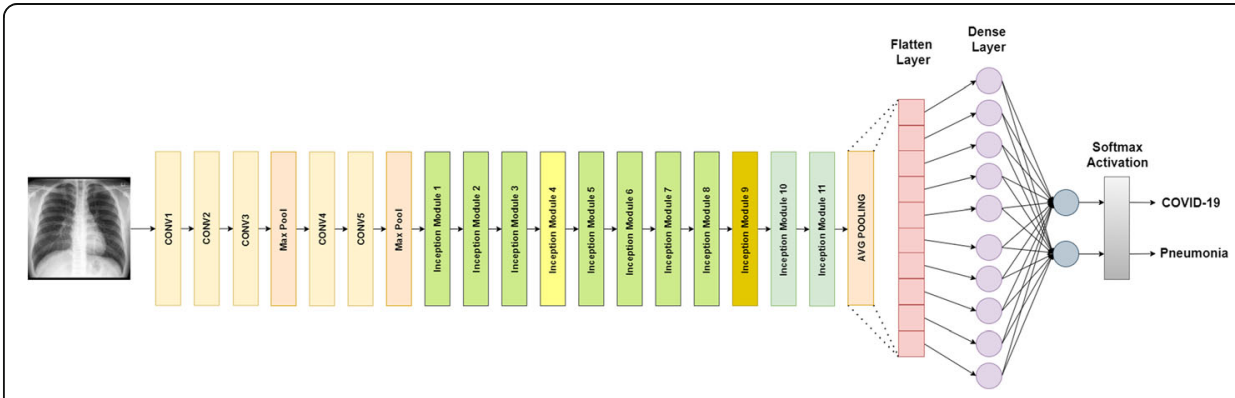

Fig. 5 InceptionV3 architecture designed for binary classification 


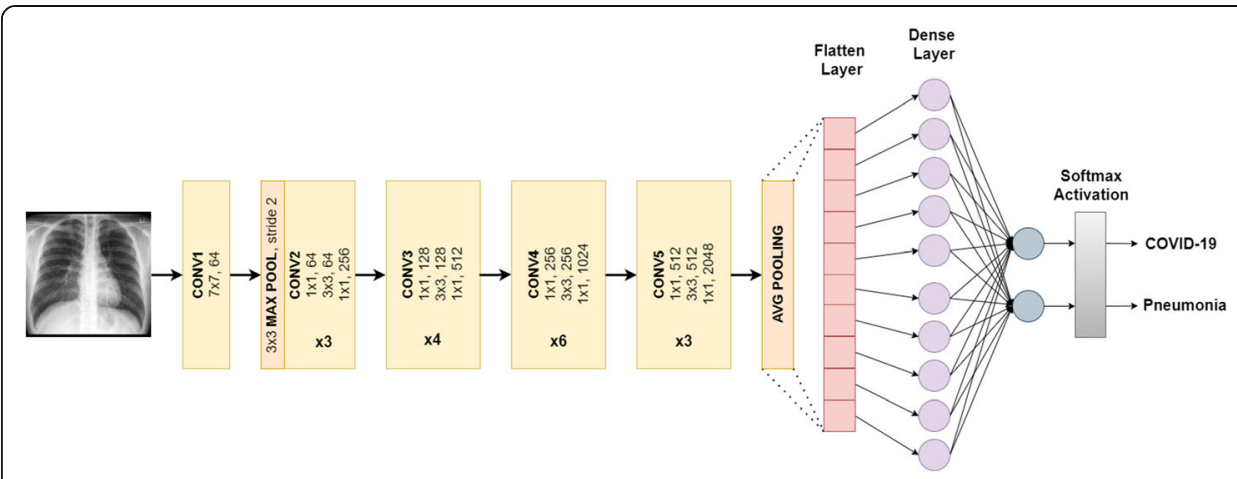

Fig. 6 ResNet50 architecture designed for binary classification

connected layer. For this study, the last fully connected layer along with softmax activation is replaced with our designed classifier as shown in Fig. 1.

\subsubsection{VGG19}

The input of VGG19 is of fixed size $224 \times 224$ RGB image. It consists of 19 layers which include 16 convolutional layers and 3 fully connected layers, including maxpooling to reduce the volume size and softmax classifier following the last fully connected layer. For this study, the last fully connected layer along with softmax activation is replaced with our designed classifier as shown in Fig. 2.

\subsubsection{DenseNet121}

The input of DenseNet121 is of fixed size $224 \times 224$ RGB image. DenseNet121 consists of 121 layers with parameters of more than 8 million. It is divided into DenseBlocks where the dimensions of the feature maps are the same within the block but the number of filters is different. The layers between the blocks are called transition layers and they apply batch normalization for down-sampling. For this study, the last fully connected layer along with softmax activation is replaced with our designed classifier as shown in Fig. 3.

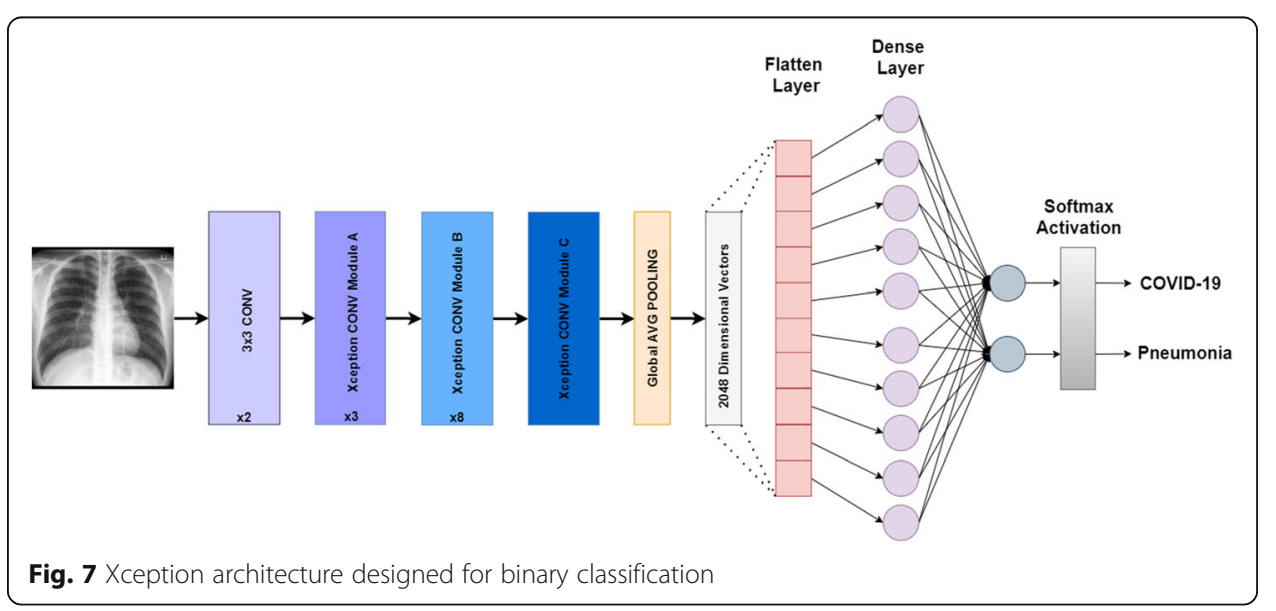


Table 3 Accuracy and loss during training and validation

\begin{tabular}{llllll}
\hline & $\begin{array}{l}\text { Best } \\
\text { epoch }\end{array}$ & $\begin{array}{l}\text { Training } \\
\text { loss }\end{array}$ & $\begin{array}{l}\text { Training } \\
\text { accuracy }\end{array}$ & $\begin{array}{l}\text { Validation } \\
\text { loss }\end{array}$ & $\begin{array}{l}\text { Validation } \\
\text { accuracy }\end{array}$ \\
\hline VGG16 & 21 & 0.0100 & 0.9990 & $0.0000 \mathrm{e}+00$ & 1.0000 \\
VGG19 & 22 & 0.0122 & 0.9994 & $0.0000 \mathrm{e}+00$ & 1.0000 \\
DenseNet121 & 20 & $6.1363 \mathrm{e}-09$ & 1.0000 & $7.2103 \mathrm{e}-07$ & 1.0000 \\
$\begin{array}{l}\text { Inception-ResNet- } \\
\text { V2 }\end{array}$ & 9 & 0.0022 & 0.9993 & 0.0011 & 1.0000 \\
InceptionV3 & 3 & 0.0472 & 0.9943 & 0.0554 & 0.9963 \\
ResNet50 & 4 & $1.1188 \mathrm{e}-04$ & 1.0000 & 0.0633 & 0.9982 \\
Xception & 4 & 0.0430 & 0.9951 & 0.0325 & 0.9926 \\
\hline
\end{tabular}

\subsubsection{Inception-ResNet-V2}

The basic building block of Inception-ResNet-V2 is called Residual Inception Block. A $1 \times 1$ convolution filter expansion layer is used after each block to scale up the filter bank dimensionality before the addition to match the depth of the input. This architecture uses batch normalization only on top of the traditional layers. Inception-ResNetV2 is 164 layers deep and has an image input size of $299 \times 299$. The Residual Inception Block incorporates multiple-sized convolutional filters with residual connections. With the use of residual connections, this architecture prevents the problem of degradation due to deep networks and reduces the duration of training. Figure 4 explains our finetuned model of Inception-ResNet-V2 for COVID-19 and pneumonia classification.

\subsubsection{InceptionV3}

InceptionV3 is made up of 484 layers consisting of 11 inception modules. It has an image input size of $299 \times 299$. Each module consists of convolution filters, pooling layers, and ReLu activation function. Without downgrading the network efficiency, InceptionV3 reduces the number of parameters by factorizing convolutions. InceptionV3 also proposed novel downsizing to reduce the number of features. Figure 5 explains our fine-tuned model of InceptionV3 for COVID-19 and pneumonia classification.

\subsubsection{ResNet50}

ResNet50 is a variant of ResNet or Residual Network. It consists of 48 convolutional layers, 1 MaxPool, and 1 average pool layer. Each convolution block has 3 convolution
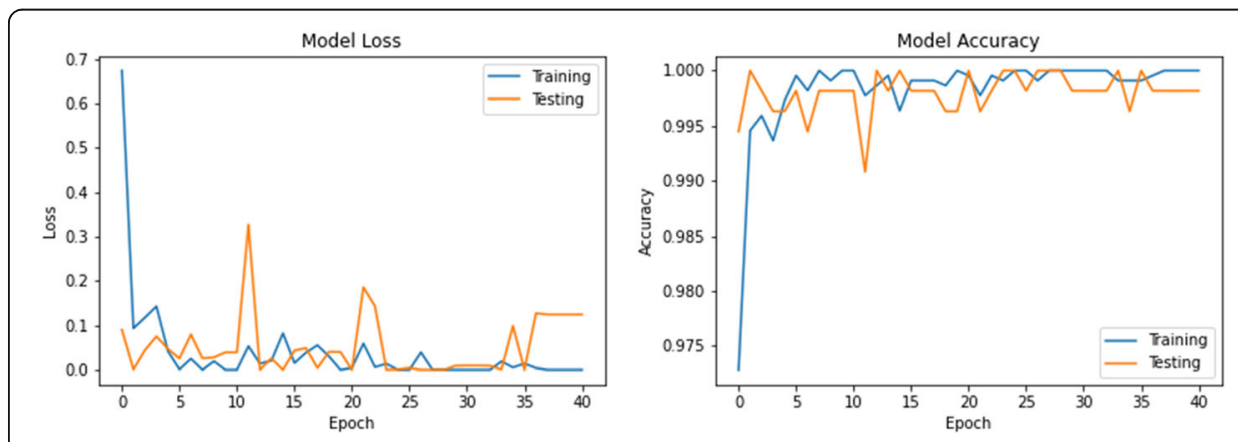

Fig. 8 VGG16 accuracy loss graph 

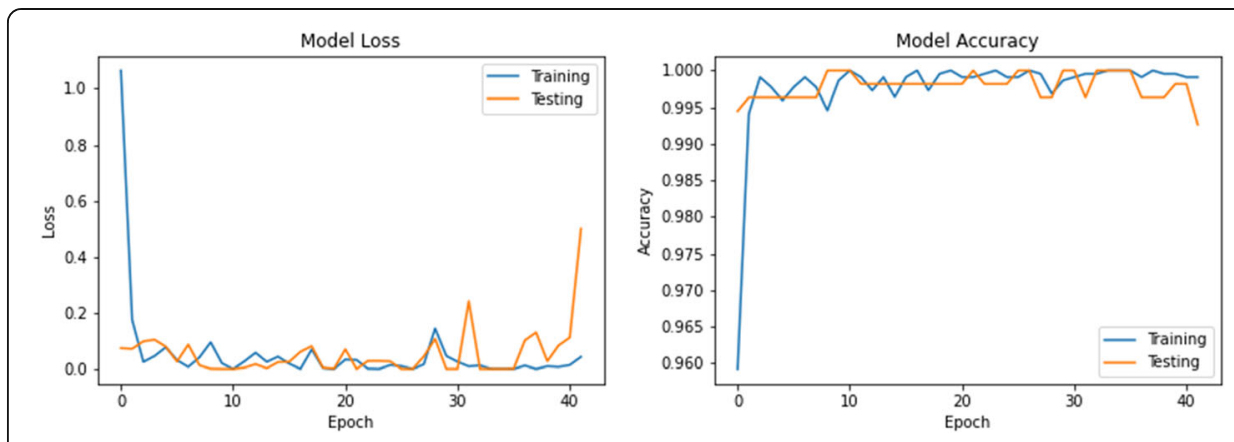

Fig. 9 VGG19 accuracy loss graph

layers, and there are also 3 convolution layers in each identification block. ResNet-50 has more than 23 million parameters which can be trained. Figure 6 explains our finetuned model of ResNet50 for COVID-19 and pneumonia classification.

\subsubsection{Xception}

Xception was proposed by Chollet in 2016, the creator of the Keras library. It is an adaption of the Inception architectures in which the Inception modules are replaced with depth-wise separable convolutions. Xception outperformed the traditional InceptionV3 with higher Top- 1 and Top- 5 accuracy on ImageNet dataset. The number of parameters of Xception is roughly the same as InceptionV3 (around 23 million). Figure 7 explains our fine-tuned model of Xception for COVID-19 and pneumonia classification.

\subsubsection{Model training}

For this study, all deep learning models-VGG16, VGG19, DenseNet121, InceptionResNet-V2, InceptionV3, Resnet50, and Xception-were trained on 12 GB NVIDIA Tesla K80 GPU. All the images of the dataset were resized to $224 \times 224$ pixels. For the algorithm development and implementation of CNN, the deep learning library - TensorFlow 2.4 with Keras API was used. The model was trained using the categorical cross-entropy loss function to measure the performance of the model from the ground truth probabilities. The categorical cross-entropy loss function is defined as:
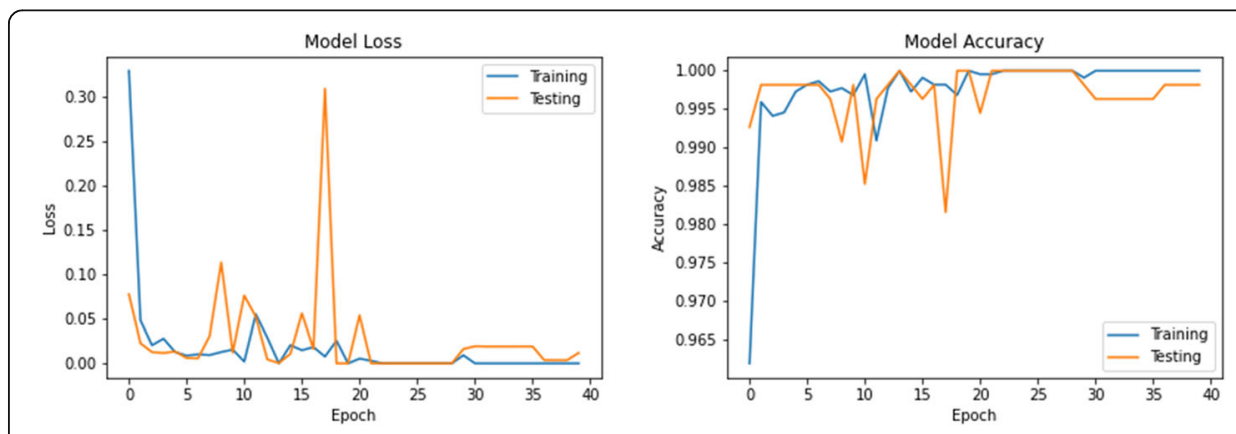

Fig. 10 DenseNet121 accuracy loss graph 

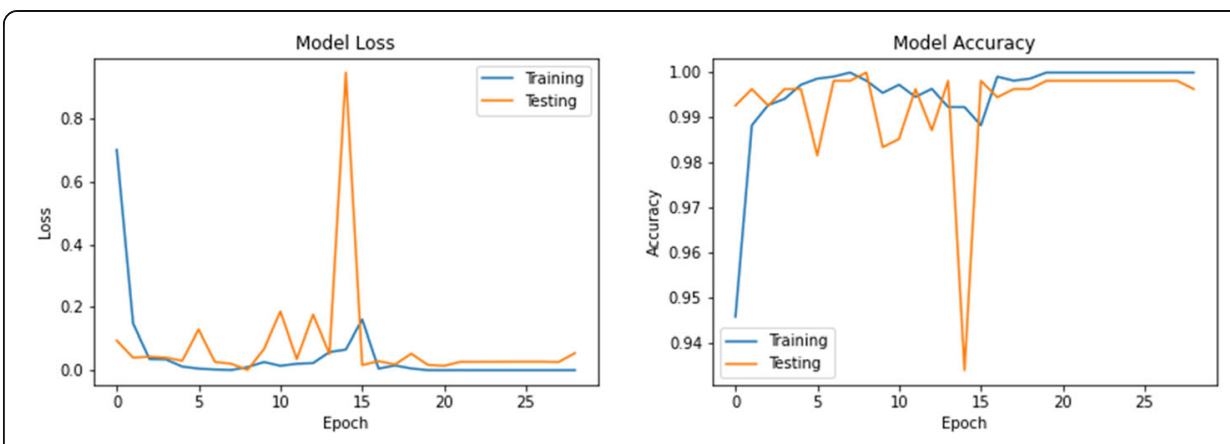

Fig. 11 Inception-ResNet-V2 accuracy loss graph

$$
\text { Categorical cross-entropy loss : }-\sum_{c=1}^{M} y_{i, c} \log \left(p_{i, c}\right)
$$

where $\mathrm{M}$ indicates the class and $y_{i, c}$ and $p_{i, c}$ indicates the ground truth and predicted probabilities for individual images. We then minimized the loss function and improved the efficacy using Adam optimizer with a learning rate of 0.001 . We implemented an early stopping technique based on validation performance to overcome the issue of overfit or underfit model. Validation loss was used as a performance measure to terminate the training when no improvement in performance was observed in 20 consecutive epochs.

\subsection{Performance metrics}

After the models finished training, they were tested on test set to evaluate the model accuracy. The models were tested on 156 COVID-19 images and 4249 pneumonia images. To evaluate the performance of the models, the metrics adopted include overall classification accuracy, recall (also known as sensitivity), precision, and F1-score. The metrics are defined as follow:

$$
\begin{aligned}
& \text { Accuracy }: \frac{\boldsymbol{T P}+\boldsymbol{T N}}{\boldsymbol{T P}+\boldsymbol{T N}+\boldsymbol{F P}+\boldsymbol{F N}} \\
& \text { Precision }: \frac{\boldsymbol{T P}}{\boldsymbol{T P}+\boldsymbol{F P}} \\
& \text { Recall }: \frac{\boldsymbol{T P}}{\boldsymbol{T P}+\boldsymbol{F N}}
\end{aligned}
$$
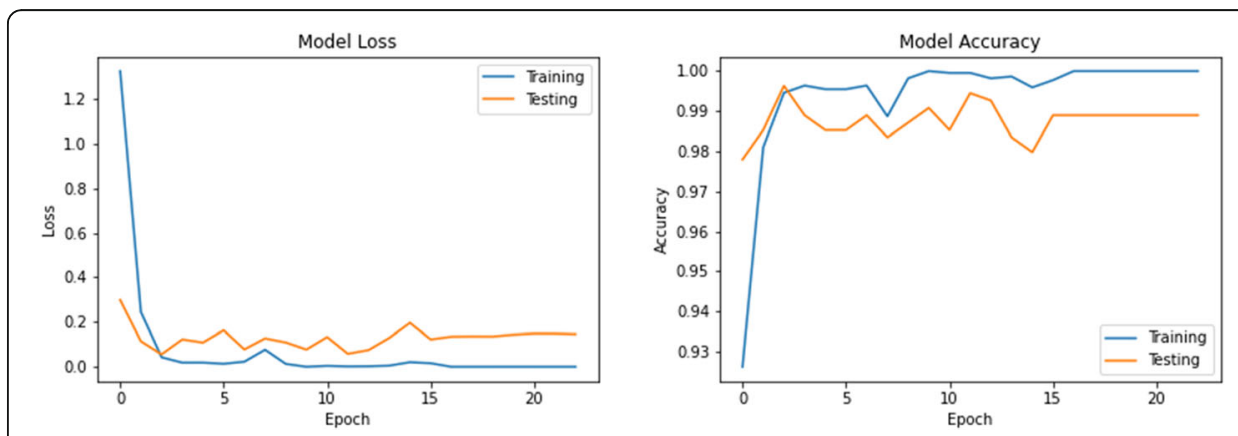

Fig. 12 InceptionV3 accuracy loss graph 

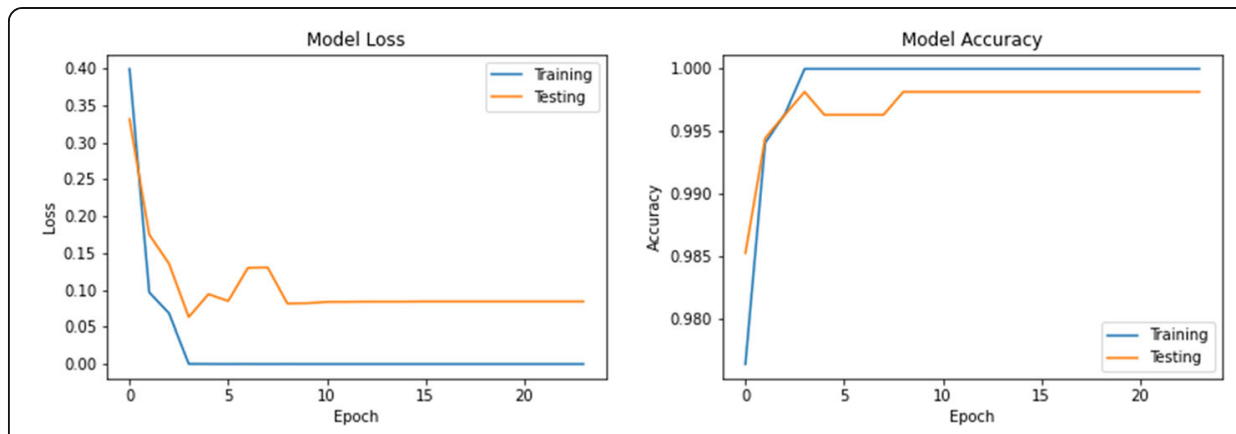

Fig. 13 ResNet50 accuracy loss graph

$$
\text { F1-score }: \frac{2 \times(\text { Recall } \times \text { Precision })}{(\text { Recall }+ \text { Precision })}
$$

where TP, TN, FP, and FN stand for true positive, true negative, false positive, and false negative. In this study, if the COVID-19 image is correctly classified, it is counted as TP, while if incorrectly classified as pneumonia, it is counted as FN. On the other hand, if a pneumonia image is classified correctly, it is counted as TN and the incorrectly classified as COVID-19 is FP. A confusion matrix was plotted to depict the number of correctly classified images, and a classification report was generated using the scikit-learn metrics function.

\section{Experimental results and discussions}

The accuracy and loss values in training and validation process are listed in Table 3 and shown in Figs. 8, 9, 10, 11, 12, 13, and 14 for each fine-tuned model. When comparing the number of epochs taken by each model to reach the minimum validation loss, it is observed that InceptionV3, ResNet50, and Xception reached a minimum loss at just 3, 4, and 4 epochs, respectively. With few epochs, they are able to achieve validation accuracy of $99 \%$ and above. This indicates that these models are able to learn the distinctive features between COVID-19 and pneumonia very quickly. However, when loss and accuracy are taken into consideration, it is observed that the training accuracy is highest for DenseNet121 and ResNet50; however, the DenseNet121 has the lowest training loss. For the validation set, VGG16, VGG19, DenseNet121, and InceptionResNet-V2 have higher accuracy; however, DenseNet121 has the lowest validation loss.
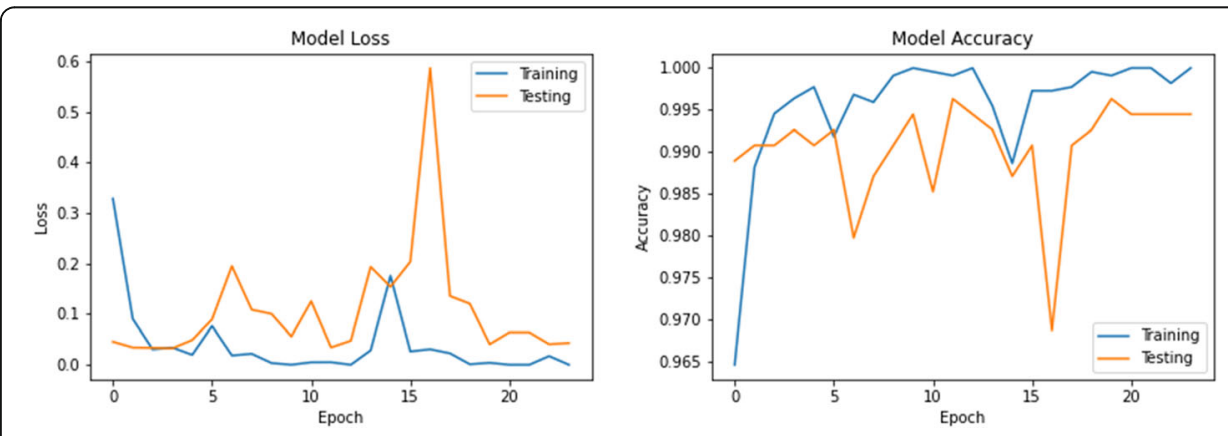

Fig. 14 Xception accuracy loss graph 
Table 4 Confusion matrix

\begin{tabular}{|c|c|c|c|c|c|c|c|c|}
\hline \multirow[t]{2}{*}{ Model } & \multicolumn{4}{|c|}{ Validation } & \multicolumn{4}{|c|}{ Testing } \\
\hline & TP & TN & FP & FN & TP & TN & FP & FN \\
\hline VGG16 & 276 & 276 & 0 & 0 & 157 & 4210 & 40 & 0 \\
\hline VGG19 & 276 & 276 & 0 & 0 & 157 & 4214 & 36 & 0 \\
\hline DenseNet121 & 276 & 276 & 0 & 0 & 156 & 4228 & 22 & 1 \\
\hline Inception-ResNet-V2 & 276 & 276 & 0 & 0 & 156 & 4172 & 78 & 1 \\
\hline InceptionV3 & 276 & 274 & 2 & 0 & 155 & 4206 & 44 & 2 \\
\hline Resnet50 & 275 & 276 & 0 & 1 & 156 & 4221 & 29 & 1 \\
\hline Xception & 274 & 274 & 2 & 2 & 155 & 4179 & 71 & 2 \\
\hline
\end{tabular}

Hence, from this data, it can be summarized that the DenseNet121 model exhibits higher training and validation performance among the seven models.

The confusion matrix displays numbers of images identified correctly and incorrectly by the model. The confusion matrix was generated for both the validation dataset and the test dataset. The validation dataset comprised of 276 COVID-19 and 276 pneumonia images whereas the test dataset comprised of 157 COVID-19 and 4250 pneumonia images. Table 4 below summarized the confusion matrix for all the seven models. It can be observed that though multiple models performed well during the validation, DenseNet121 has the lowest false positive and false negative, indicating that the DenseNet121 model, as shown in Fig. 15, made the least number of errors while predicting the image was COVID-19 or pneumonia.

This study even compared these pre-trained models based on the accuracy, precision, recall, and F1 score as tabulated in Table 5. It is observed that DenseNet121 gave good classification performance with an accuracy of $99.48 \%$, followed by ResNet50 with 99.32\% accuracy. Table 5 also compares the computational times taken by each model for training and testing. It is seen that the InceptionV3 takes the least time (11 min 50 s) for training; however, it is slow during testing ( 16 min $14 \mathrm{~s})$, whereas DenseNet121 was slower during training ( $20 \mathrm{~min}$ ), but it was the fastest during testing (15 min $36 \mathrm{~s}$ ) with the highest accuracy.

From the above result, we recommend DenseNet121 (99.48\% accuracy, 99.54\% precision, $99.48 \%$ recall, and $99.49 \%$ of F1 score) for classification of COVID-19

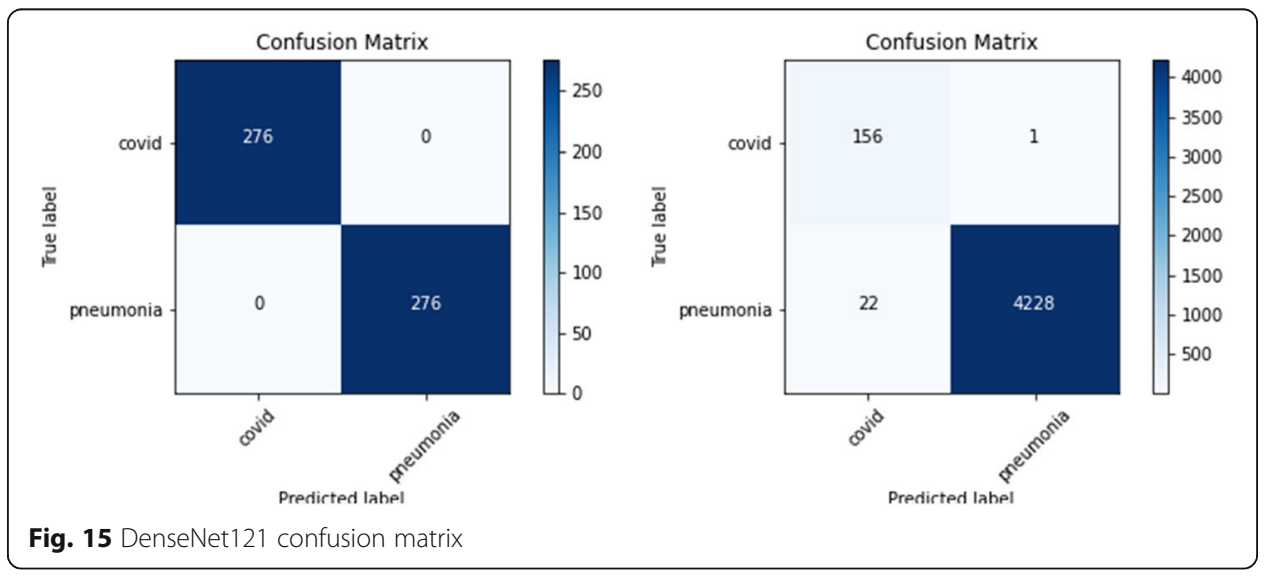


Table 5 Performance metrics

\begin{tabular}{lllllll}
\hline Model & $\begin{array}{l}\text { Accuracy } \\
(\%)\end{array}$ & $\begin{array}{l}\text { Precision } \\
(\%)\end{array}$ & $\begin{array}{l}\text { Recall } \\
(\%)\end{array}$ & $\begin{array}{l}\text { F1 } \\
(\%)\end{array}$ & $\begin{array}{l}\text { Training time } \\
\text { (s) }\end{array}$ & $\begin{array}{l}\text { Testing time } \\
\text { (s) }\end{array}$ \\
\hline VGG16 & 99.09 & 99.28 & 99.09 & 99.14 & 1353 & 1012 \\
VGG19 & 99.18 & 99.34 & 99.18 & 99.22 & 1344 & 946 \\
DenseNet121 & 99.48 & 99.54 & 99.48 & 99.49 & 1200 & 936 \\
$\begin{array}{l}\text { Inception-ResNet- } \\
\text { V2 }\end{array}$ & 98.21 & 98.79 & 98.21 & 98.38 & 899 & 1083 \\
InceptionV3 & 98.96 & 99.17 & 98.96 & 99.02 & 710 & 974 \\
ResNet50 & 99.32 & 99.42 & 99.32 & 99.35 & 783 & 1056 \\
Xception & 98.34 & 98.83 & 98.34 & 98.49 & 750 & 865 \\
\hline
\end{tabular}

from pneumonia cases on chest X-ray, further comparing our fine-tuned DenseNet121 with the studies recently published that also performed binary classification, particularly COVID-19 and pneumonia images. Shelke et al. [15] used a deeper network, DenseNet-161, but the accuracy obtained is lower, which might be due to the lower number of training images. Compared with other works that worked on the binary classification of CXR images, our model has the second highest accuracy (Table 6). The highest binary classification accuracy is obtained by Gupta et al. [19] using their proposed network called InstaCovNet-19.

\section{Conclusion}

Deep learning algorithm can aid healthcare workers in detecting COVID-19 with minimal processing of chest X-ray images. In this study, 2-class datasets were created which included COVID-19 and pneumonia images obtained from open

Table 6 Comparison with other related work that performed binary classification on CXR images

\begin{tabular}{|c|c|c|c|c|}
\hline $\begin{array}{l}\text { Author } \\
\text { (year) }\end{array}$ & CNN architectures & $\begin{array}{l}\text { Image } \\
\text { classification }\end{array}$ & $\begin{array}{l}\text { Total } \\
\text { dataset }\end{array}$ & Accuracy \\
\hline Nayak et al. [37] & ResNet-34 & COVID-19 and normal & $\begin{array}{l}\text { COVID-19: } 775 \\
\text { Normal: } 775\end{array}$ & $98.33 \%$ \\
\hline $\begin{array}{l}\text { Ismael and Şengür } \\
\text { [11] }\end{array}$ & ResNet50 feature + SVM & COVID-19 and normal & $\begin{array}{l}\text { COVID-19: } 180 \\
\text { Normal: } 200\end{array}$ & $94.7 \%$ \\
\hline Ozturk et al. [14] & DarkCovidNet & COVID-19 and no-findings & $\begin{array}{l}\text { COVID-19: } 177 \\
\text { No-findings: } 500\end{array}$ & $98.08 \%$ \\
\hline $\begin{array}{l}\text { Amit Kumar et al. } \\
\text { [38] }\end{array}$ & $\begin{array}{l}\text { Ensembling method: three } \\
\text { pretrained CNN models- } \\
\text { DenseNet201, Resnet50V2, } \\
\text { and Inceptionv3 }\end{array}$ & $\begin{array}{l}\text { COVID-19 positive and } \\
\text { COVID-19 negative }\end{array}$ & $\begin{array}{l}\text { COVID-19 +ve: } \\
538 \\
\text { COVID-19 -ve: } \\
468\end{array}$ & $95.7 \%$ \\
\hline Gupta et al. [19] & $\begin{array}{l}\text { InstaCovNet-19 (integrated } \\
\text { stacking of InceptionV3, } \\
\text { NASnet, Xception, MobileNetV2 } \\
\text { and ResNet101) }\end{array}$ & COVID-19 and non-COVID & $\begin{array}{l}\text { COVID-19: } 361 \\
\text { Normal: } 365\end{array}$ & $99.53 \%$ \\
\hline Hussain et al. [20] & CoroDet & COVID-19 and normal & $\begin{array}{l}\text { COVID-19: } 2843 \\
\text { Normal: } 1108\end{array}$ & $99.1 \%$ \\
\hline Shelke et al. [15] & DenseNet-161 & COVID-19 and pneumonia & $\begin{array}{l}\text { COVID-19: } 500 \\
\text { Pneumonia: } 500\end{array}$ & $98.9 \%$ \\
\hline Jain et al. [9] & ResNet-101 & $\begin{array}{l}\text { COVID-19 and viral } \\
\text { pneumonia }\end{array}$ & $\begin{array}{l}\text { COVID-19: } 440 \\
\text { Viral pneumonia: } \\
480\end{array}$ & $97.78 \%$ \\
\hline This study & DenseNet-121 & $\begin{array}{l}\text { COVID-19 and } \\
\text { pneumonia }\end{array}$ & $\begin{array}{l}\text { COVID-19: } 1536 \\
\text { Pneumonia: } \\
5629\end{array}$ & $99.48 \%$ \\
\hline
\end{tabular}


sources. Several state-of-the-art pretrained neural networks that include ResNet50, DenseNet121, InceptionV3, VGG16, VGG19, Inception-ResNet-V2, and Xception were experimented using transfer learning technique. The best model turned out to be DenseNet-121 which accomplished an accuracy of $99.48 \%$, followed by ResNet50 with a classification accuracy of $99.32 \%$. This study summarizes that the detection models built using CNNs with transfer learning technique are able to perform good binary classification tasks on COVID-19 and pneumonia images. COVID-19 and viral pneumonia CXR images contain similar features which are challenging for the radiologist to interpret. However, the CNN model can easily learn the features in just a few epochs of training and classify the images correctly. The high accuracies obtained suggest that the deep learning models could find something distinctive in the CXR images and that makes the deep networks capable of distinguishing the images correctly. These trained models can effectively reduce the workload of medical practitioners and increase the accuracy and efficiency of COVID-19 diagnosis.

\begin{abstract}
Abbreviations
CNN: Convolutional neural network; COVID-19: Coronavirus disease of 2019; CT: Computed tomography; CXR: Chest Xray; DeTraC: Decompose, Transfer, and Compose; DNA: Deoxyribonucleic acid; FFT: Fast Fourier transform; FN: False negatives; FP: False positives; GLCM: Gray-Level Co-Occurrence Matrix; GLDM: Gray Level Dependence Matrix; RNA: Ribonucleic acid; rRT-PCR: Real-time reverse transcription-polymerase chain reaction; SARS: Severe acute respiratory syndrome; SVM: Support vector machine; TN: True negatives; TP: True positive; VGG: Visual Geometry Group
\end{abstract}

\title{
Acknowledgements
}

This work was supported by the 2020 EBC-C (Extra-Budgetary Contributions from China) Project on Promoting the Use of ICT for Achievement of Sustainable Development Goals (IF015-2021), and University Malaya.

\section{Authors' contributions}

All the authors contributing equally in data collection, processing, experiments, and article writing. The authors read and approved the final manuscript.

\author{
Authors' information \\ Shazia Anis \\ Received her degree in Biomedical Engineering from Ajman University (UAE) in 2015. Master in Biomedical \\ Engineering from the University of Malaya in 2018. She is currently pursuing her PhD in Biomedical Engineering from \\ the University of Malaya. Her research interests include biomedical imaging, machine learning, and image processing. \\ Zi Xuan Tan \\ He is currently in his final year of study, pursuing the bachelor's degree of Biomedical Engineering at Universiti Malaya. \\ His research interests include machine learning and medical image processing. \\ Joon Huang Chuah \\ He received the B.Eng. (Hons.) degree from the Universiti Teknologi Malaysia, the M.Eng. degree from the National \\ University of Singapore, and the M.Phil. and Ph.D. degrees from the University of Cambridge. He is currently Head of \\ VIP Research Group and a Senior Lecturer at the Department of Electrical Engineering, Faculty of Engineering, \\ University of Malaya. He is a Chartered Engineer registered under the Engineering Council, UK, and also a Professional \\ Engineer registered under the Board of Engineers, Malaysia. He is the Honorary Treasurer of IEEE Computational \\ Intelligence Society (CIS) Malaysia Chapter and the Honorary Secretary of IEEE Council on RFID Malaysia Chapter. He is \\ also the Honorary Treasurer of the Institution of Engineering and Technology (IET) Malaysia Network. He is a Fellow \\ and the Honorary Secretary of the Institution of Engineers, Malaysia (IEM). His main research interests include image \\ processing, computational intelligence, IC design, and scanning electron microscopy. \\ Juliana Usman \\ Dr. Juliana Usman is a Senior Lecturer from the Department of Biomedical Engineering, University of Malaya. She \\ graduated with a Bachelor of Biomedical Engineering degree and a Masters of Engineering Science from the \\ University of Malaya. She received her doctorate degree from the University of New South Wales in 2012. Her area of \\ interest is sports biomechanics specializing in injury prevention and performance enhancement. \\ Pengjiang Qian \\ Pengjiang Qian received the B.S. degree in computer science and technology from Jiangnan University, Wuxi, China, in \\ 2000, the M.S. degree in software engineering from the Nanjing University of Science and Technology, Nanjing, China, \\ in 2005, and the Ph.D. degree in information technology and engineering from Jiangnan University, in 2011. He is an \\ Associate Professor with the School of Digital Media, Jiangnan University. He is currently with the Case Western \\ Reserve University, Cleveland, OH, USA, as a Visiting Scholar and doing research in medical image processing. He has \\ published nearly 30 papers in international/national journals and conferences. His current research interests include \\ data mining, pattern recognition, bioinformatics, and their applications, such as medical image processing. \\ Khin Wee Lai
}


Received his PhD from Technische Universitat IImenau, Germany, and Universiti Teknologi Malaysia (UTM) under DAAD $\mathrm{PhD}$ Sandwich Programme. He is currently the Head Programme - Master of Engineering (Biomedical) at Faculty of Engineering, University Malaya. He is a Chartered Engineer registered under the Engineering Council, U.K., Engineers Australia, APEC Engineer, IntPE (Australia), and also a Professional Engineer registered under the Board of Engineers, Malaysia. He is a Fellow of Engineers, Australia, and a Fellow of the Institution of Engineers, Malaysia (IEM). His research interests include computer vision, machine learning, medical image processing, and healthcare analytics.

\section{Funding}

This work was supported by the 2020 EBC-C (Extra-Budgetary Contributions from China) Project on Promoting the Use of ICT for Achievement of Sustainable Development Goals (IF015-2021), and University of Malaya.

\section{Availability of data and materials}

All the data are available upon request from the corresponding author.

\section{Declarations}

Ethics approval and consent to participate

Not applicable. All the databases were obtained from the literature that are publicly available.

\section{Consent for publication}

Not applicable.

\section{Competing interests}

There is no conflict of interest reported by any authors.

\section{Author details}

${ }^{1}$ Department of Biomedical Engineering, Faculty of Engineering, Universiti Malaya, 50603 Kuala Lumpur, Malaysia. ${ }^{2}$ Department of Electrical Engineering, Faculty of Engineering, Universiti Malaya, 50603 Kuala Lumpur, Malaysia. ${ }^{3}$ School of Artificial Intelligence and Computer Science, Jiangnan University, 1800 Lihu Avenue, Jiangsu 214122 Wuxi, People's Republic of China.

\section{Received: 13 April 2021 Accepted: 23 June 2021}

Published online: 27 July 2021

\section{References}

1. V.M. Corman et al., Detection of 2019 novel coronavirus (2019-nCoV) by real-time RT-PCR. Euro. Surveill. 25(3), 2000045 (2020). https://doi.org/10.2807/1560-7917.ES.2020.25.3.2000045

2. Y. Fang et al., Sensitivity of Chest CT for COVID-19: comparison to RT-PCR. Radiology 296(2), E115-E117 (2020). https:// doi.org/10.1148/radiol.2020200432

3. T.C. Williams et al., Sensitivity of RT-PCR testing of upper respiratory tract samples for SARS-CoV-2 in hospitalised patients: a retrospective cohort study. medRxiv (2020). https://doi.org/10.1101/2020.06.19.20135756

4. J.P. Kanne, B.P. Little, J.H. Chung, B.M. Elicker, L.H. Ketai, Essentials for radiologists on COVID-19: An Update-Radiology Scientific Expert Panel. Radiology 296(2), E113-E114 (2020). https://doi.org/10.1148/radiol.2020200527

5. J.F.-W. Chan et al., A familial cluster of pneumonia associated with the 2019 novel coronavirus indicating person-toperson transmission: a study of a family cluster. Lancet 395(10223), 514-523 (2020). https://doi.org/10.1016/s0140-6736(2 0)30154-9

6. L.A. Rousan, E. Elobeid, M. Karrar, Y. Khader, Chest x-ray findings and temporal lung changes in patients with COVID-19 pneumonia. BMC Pulmonary Med. 20(1), 245 (2020). https://doi.org/10.1186/s12890-020-01286-5

7. S. Asif, Y. Wenhui, H. Jin, Y. Tao, S. Jinhai, Classification of COVID-19 from chest X-ray images using deep convolutional neural networks. medRxiv (2020). https://doi.org/10.1101/2020.05.01.20088211

8. S. Anis et al., An overview of deep learning approaches in chest radiograph. IEEE Access 8, 182347-182354 (2020). https://doi.org/10.1109/ACCESS.2020.3028390

9. G. Jain, D. Mittal, D. Thakur, M.K. Mittal, A deep learning approach to detect Covid-19 coronavirus with X-Ray images. Biocybern. Biomed. Eng. 40(4), 1391-1405 (2020). https://doi.org/10.1016/j.bbe.2020.08.008

10. M.Z. Che Azemin, R. Hassan, M.I. Mohd Tamrin, M.A. Md Ali, COVID-19 deep learning prediction model using publicly available radiologist-adjudicated chest X-ray images as training data: preliminary findings. Int. J. Biomed. Imaging, 8828855 (2020, 2020). https://doi.org/10.1155/2020/8828855

11. A.M. Ismael, A. Şengür, Deep learning approaches for COVID-19 detection based on chest X-ray images. Expert Syst. Appl. 164, 114054 (2021). https://doi.org/10.1016/j.eswa.2020.114054

12. A. Makris, I. Kontopoulos, K. Tserpes, COVID-19 detection from chest X-ray images using deep learning and convolutional neural networks. medRxiv (2020). https://doi.org/10.1101/2020.05.22.20110817

13. A. Abbas, M.M. Abdelsamea, M.M. Gaber, Classification of COVID-19 in chest X-ray images using DeTraC deep convolutional neural network. Appl. Intell. 51(2), 854-864 (2021). https://doi.org/10.1007/s10489-020-01829-7

14. T. Ozturk, M. Talo, E.A. Yildirim, U.B. Baloglu, O. Yildirim, U. Rajendra Acharya, Automated detection of COVID-19 cases using deep neural networks with X-ray images. Comput. Biol. Med. 121, 103792-103792 (2020). https://doi.org/10.1016/j. compbiomed.2020.103792

15. A. Shelke et al., Chest X-ray classification using deep learning for automated COVID-19 screening. medRxiv (2020). https://doi.org/10.1101/2020.06.21.20136598

16. S. Minaee, R. Kafieh, M. Sonka, S. Yazdani, G. Jamalipour Soufi, Deep-COVID: Predicting COVID-19 from chest X-ray images using deep transfer learning. Med Image Anal 65, 101794 (2020). https://doi.org/10.1016/j.media.2020.101794 
17. A. K. Das, S. Ghosh, S. Thunder, R. Dutta, S. Agarwal, and A. Chakrabarti, "Automatic COVID-19 detection from X-ray images using ensemble learning with convolutional neural network," Pattern Analysis and Applications. 2021/03/19 2021. https://doi.org/10.1007/s10044-021-00970-4.

18. A. Ridhi et al., Al-based Diagnosis of COVID-19 Patients Using X-ray Scans with Stochastic Ensemble of CNNs. 2020.

19. A. Gupta, S.G. Anjum, R. Katarya, InstaCovNet-19: A deep learning classification model for the detection of COVID-19 patients using Chest X-ray. Appl. Soft Comput. 99, 106859 (2021). https://doi.org/10.1016/j.asoc.2020.106859

20. E. Hussain, M. Hasan, M.A. Rahman, I. Lee, T. Tamanna, M.Z. Parvez, CoroDet: A deep learning based classification for COVID-19 detection using chest X-ray images. Chaos Solitons Fractals 142, 110495 (2021). https://doi.org/10.1016/j.cha os.2020.110495

21. M. Canayaz, MH-COVIDNet: Diagnosis of COVID-19 using deep neural networks and meta-heuristic-based feature selection on X-ray images. Biomed. Signal Process. Control 64, 102257 (2021). https://doi.org/10.1016/j.bspc.2020.102257

22. A.Z. Khuzani, M. Heidari, S.A. Shariati, COVID-Classifier: An automated machine learning model to assist in the diagnosis of COVID-19 infection in chest x-ray images. medRxiv (2020). https://doi.org/10.1101/2020.05.09.20096560

23. M.F. Hashmi, S. Katiyar, A.G. Keskar, N.D. Bokde, Z.W. Geem, Efficient pneumonia detection in chest Xray images using deep transfer learning. Diagnostics 10(6), 417 (2020) [Online]. Available: https://www.mdpi.com/2075-4418/10/6/417

24. J.R. Zech, M.A. Badgeley, M. Liu, A.B. Costa, J.J. Titano, E.K. Oermann, Variable generalization performance of a deep learning model to detect pneumonia in chest radiographs: a cross-sectional study. Plos Med 15(11), e1002683 (2018). https://doi.org/10.1371/journal.pmed.1002683

25. E. Ayan, H.M. Ünver, in 2019 Scientific Meeting on Electrical-Electronics \& Biomedical Engineering and Computer Science (EBBT). Diagnosis of pneumonia from chest X-ray images using deep learning (2019), pp. 1-5. https://doi.org/10.1109/ EBBT.2019.8741582

26. A. Narin, C. Kaya, Z. Pamuk, Automatic detection of coronavirus disease (COVID-19) using X-ray images and deep convolutional neural networks. Pattern Anal. Appl. (2021). https://doi.org/10.1007/s10044-021-00984-y

27. I.U. Khan, N. Aslam, A deep-learning-based framework for automated diagnosis of COVID-19 using X-ray images. Information 11(9), 419 (2020) [Online]. Available: https://www.mdpi.com/2078-2489/11/9/419

28. Praveen. CoronaHack -Chest X-Ray-Dataset. [Online]. Available: https://www.kaggle.com/praveengovi/coronahack-chestxraydataset. Accessed 21 Mar 2020.

29. Z. Li. Run some Covid-19 lung X-Ray classification and CT detection demos [Online] Available: https://community. intersystems.com/post/run-some-covid-19-lung-X-ray-classification-and-ct-detection-demos. Accessed 16 Apr 2020

30. T. Rahman, M. Chowdhury, and A. Khandakar. COVID-19 Radiography Database. [Online]. Available: https://www.kaggle. com/tawsifurrahman/covid19-radiography-database. Accessed 29 Mar 2020.

31. J. P. Cohen. covid-chestxray-dataset. [Online]. Available: https://github.com/ieee8023/covid-chestxray-dataset. Accessed 02 Oct 2020

32. A. Chung. Figure1-COVID-chestxray-dataset. [Online]. Available: https://github.com/agchung/Figure1-COVID-chestxray-da taset. Accessed 09 May 2020.

33. A. Chung. Actualmed-COVID-chestxray-dataset. [Online]. Available: https://github.com/agchung/Actualmed-COVIDchestxray-dataset. Accessed 06 May 2020.

34. N. Sajid. COVID-19 Patients Lungs X Ray Images 10000. [Online]. Available: https://www.kaggle.com/nabeelsajid917/ covid-19-x-ray-10000-images. Accessed 24 Mar 2020.

35. Kermany, Daniel; Zhang, Kang; Goldbaum, Michael (2018), "Labeled Optical Coherence Tomography (OCT) and Chest XRay Images for Classification", Mendeley Data, V2. https://doi.org/10.17632/rscbjbr9sj.2. Accessed 25 Mar 2020.

36. R.L.D.J. Qiong Wei, The role of balanced training and testing data sets for binary classifiers in bioinformatics. Plos One (2013). https://doi.org/10.1371/journal.pone.0067863

37. S.R. Nayak, D.R. Nayak, U. Sinha, V. Arora, R.B. Pachori, Application of deep learning techniques for detection of COVID-19 cases using chest X-ray images: a comprehensive study. Biomed. Signal Process. Control 64, 102365 (2021). https://doi. org/10.1016/j.bspc.2020.102365

38. D. Amit Kumar, G. Sayantani, T. Samiruddin, D. Rohit, A. Sachin, C. Amlan, Automatic COVID-19 detection from X-ray images using ensemble learning with convolutional neural network. Res. Square (2020). https://doi.org/10.21203/rs.3.rs51360/v1

\section{Publisher's Note}

Springer Nature remains neutral with regard to jurisdictional claims in published maps and institutional affiliations.

\section{Submit your manuscript to a SpringerOpen ${ }^{\circ}$ journal and benefit from:}

- Convenient online submission

- Rigorous peer review

- Open access: articles freely available online

- High visibility within the field

- Retaining the copyright to your article

Submit your next manuscript at $\boldsymbol{\Delta}$ springeropen.com 